\title{
Improvement and Performance Evaluation of CAVENET: A Network Simulation Tool for Vehicular Networks
}

\author{
Leonard Barolli*, Gjergji Mino ${ }^{\dagger}$, Fatos Xhafa ${ }^{\ddagger}$ Arjan Durresi $^{\S}$ and Akio Koyama \\ * Department of Information and Communication Engineering \\ Fukuoka Institute of Technology (FIT) \\ 3-30-1 Wajiro-Higashi, Higashi-Ku, Fukuoka 811-0295, Japan \\ E-mail: barolli@fit.ac.jp \\ ${ }^{\dagger}$ Graduate School of Engineering \\ Fukuoka Institute of Technology (FIT) \\ 3-30-1 Wajiro-Higashi, Higashi-Ku, Fukuoka 811-0295, Japan \\ E-mail: bd09002@bene.fit.ac.jp \\ $\ddagger$ Technical University of Catalonia \\ Department of Languages and Informatics Systems \\ C/Jordi Girona 1-3, 08034 Barcelona, Spain \\ E-mail:fatos@lsi.upc.edu \\ $\S$ Department of Computer and Information Science \\ Indiana University Purdue University at Indianapolis (IUPUI) \\ 723 W. Michigan Street SL 280 Indianapolis, IN 46202, USA \\ E-mail: durresi@cs.iupui.edu \\ "Department of Informatics \\ Graduate School of Science and Engineering \\ Yamagata University \\ 4-3-16 Jonan, Yonezawa 992-8510, Yamagata, Japan \\ E-mail: akoyama@yz.yamagata-u.ac.jp
}

\begin{abstract}
Vehicle Ad-hoc Network (VANET) is a kind of Mobile Ad-hoc Network (MANET) that establishes wireless connection between cars. In VANETs and MANETs, the topology of the network changes very often, therefore implementation of efficient routing protocols is very important problem. In MANETs, the Random Waypoint (RW) model is used as a simulation model for generating node mobility pattern. On the other hand, in VANETs, the mobility patterns of nodes is restricted along the roads, and is affected by the movement of neighbour nodes. In this paper, we present a simulation system for VANET called CAVENET (Cellular Automaton based VEhicular NETwork). In CAVENET, the mobility patterns of nodes are generated by an 1-dimensional cellular automata. We improved CAVENET and implemented some routing protocols. We investigated the performance of the implemented routing protocols by CAVENET. The simulation results have shown that DYMO protocol has better performance than AODV and OLSR protocols.
\end{abstract}

Keywords-VANET; MANET; CAVENET; Ad-Hoc Networks Protocols.

\section{INTRODUCTION}

Vehicular communication is seen as a key technology for improving road safety and comfort through Intelligent Transportation Systems (ITS). There are many possible application of wireless technologies for vehicular environment [1].
Vehicular Ad Hoc Networks (VANETs) are an instance of ad-hoc networks, which are general-purpose distributed wireless networks interconnected without the need of any centralized infrastructure. VANETs are expected to be massively deployed in upcoming vehicles, because their use can improve the safety of driving and makes new forms of inter-vehicle communications possible as well. Given a mobility model of vehicles, usually a simulator is used to test networking protocols. In this regard, we present a lightweight simulator which can be used to understand the properties of the mobility models of vehicular traffic and their impact on the performance of VANETs. We call this simulator Cellular Automaton based VEhicular NETwork (CAVENET), because its mobility model is built upon a 1dimensional Cellular Automaton (CA).

The CAVENET separates the problem of mobility model from that of the protocol evaluation, which is performed by means of a network simulator. The properties of the mobility model, e.g. the average transient time towards the stationary state, can be analysed independently of the protocol simulation. Eventually, the movement patterns generated by the mobility model can be mapped into a trace file format suitable for the network simulator. 
The Random Waypoint (RW) model has been the earliest mobility model for ad-hoc networks. Basically, in RW every node picks up a random destination and a random velocity at certain points called waypoints. This model has been extended in a number of ways in order to take into account more realistic movements. The simulation of such models has shown the problem of velocity decay, which posed some doubts about the length of the simulation time and the duration of the transient. The problem has been solved by several authors, in particular by Le Boudec [2], who used Palm distributions, and Noble [3]. However, all mobility models considered so far are Short Range Dependent (SRD). This means that every mobile chooses its velocity independently by the others. In the case of VANETs, this assumption is clearly not valid anymore, especially in the case of highway traffic. We show this fact by means of basic simulations performed with CAVENET. For instance, we show that the traffic model strongly affects the statistical structure of the average velocity.

In the particular case of deterministic traffic models, the average velocity is SRD and the transient state depends on the density of the vehicles. In general, the mobility model of VANETs for the simulated variable of interest (e.g. the average velocity) can be Long Range Dependent (LRD) in some cases. This fact poses some problems on how long the simulation should be and how many samples from the starting time should be discarded.

In literature, vehicular mobility models are usually classified as either macroscopic or microscopic. The macroscopic description models gross quantities of interest, such as vehicular density or mean velocity, treating vehicular traffic according to fluid dynamics, while the microscopic description considers each vehicle as a distinct entity, modelling its behaviour in a more precise, but computationally more expensive way. Yet, a micro-macro approach may be seen more as a broad classification schema than a formal description of the models' functionalities in each class [1].

In this work, we consider the vehicular mobility model as a microscopic model. Our simulator is based on 1dimensional CA model. The CA is a discrete time model of the vehicular traffic. The first version of CAVENET had some problems. For this reason, we improved the CAVENET, by changing the movement pattern of the vehicles from the straight line to a circle. We also implemented three routing protocols for Ad Hoc networks: Optimized Link State Routing (OLSR) [4], Ad-hoc On-demand Distance Vector (AODV) [5], Dynamic MANET On Demand (DYMO) [6], [7], and investigated their performance for VANETs.

The rest of the paper is structured as follows. In Section II, we discuss the related work for VANETs. In Section III, we present CAVENET structure and description. In Section IV, we discuss the simulation results. Finally, the conclusions and future work are presented in Section V.

\section{RELATED WORK}

In general, a simulator should have the following properties.

1) It should be open source, in order to let other users criticize the validity of the model and the implementation.

2) The code should be clear, in order to let others performing the task in 1 .

3) The structure should be modular, in order to analyse single pieces of the simulation process.

In the recent years, a lot of simulators for VANETs have been emerging [1]. For example, the IMPORTANT framework has been one of the first attempt to understand the dependence between vehicular traffic and communication performance [8], [9]. The authors analyzed the impact of the node mobility on the duration of communication paths. However, the author implemented the code in $\mathrm{C}$, which is difficult to debug and extend without the support of a detailed documentation. Moreover, it seems that their Freeway model is not as realistic as the model we study here.

In [10], the authors present a simulator written in Java, which can generate mobility traces in several formats. The details of the implementation are not open. There are also other powerful traffic simulators, like TranSim [11], which makes use of a cellular automaton for simulating the interaction of vehicles. Unfortunately, the code is not conceived for network protocols simulation, and the software is commercially licensed. Also, SUMO is another powerful traffic simulator, intended for traffic planning and road design optimization. There is an attempt to interface SUMO with ns-2 [12]. However, in our opinion, it is very expensive to understand the SUMO language and also unnecessary, because the communications engineer needs only a parsimonious model, easy to extend and/or modify.

There are many other works which consider the possibility of using ad-hoc and MANET protocols for VANET scenarios. A car taking part in a MANET scenario could establish connections using the public hotspots while driving in the city. Also, the deployment of access points along highways in the near future seems feasible. Thus, it is important to investigate the application of MANET routing protocols for VANETs [13], [14], [15]. In [13], the authors present only the way of generating the vehicle movement pattern. They did not evaluate the performance of routing protocols. While, in [14], the authors used the simulation in [15] and present the performance evaluation for AODV and OLSR protocols. However, they use the uniform distribution for the generation of the node movement.

\section{CAVENET STRUCTURE AND DESCRIPTION}

The mobility model for VANETs should take into account the following parameters. 


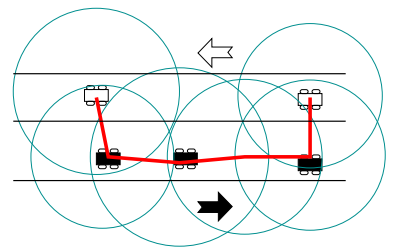

(a)

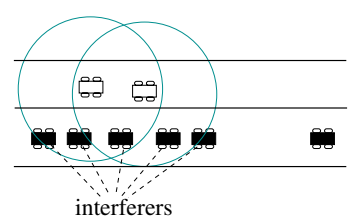

(b)
Figure 1. Impact of multi-lanes: a) on the connectivity; b) on the interference.

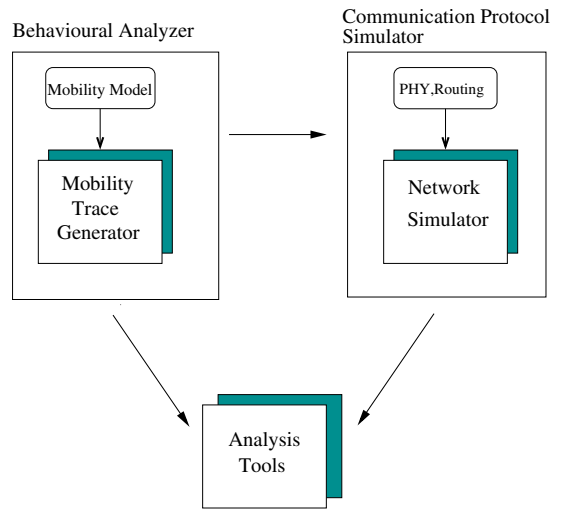

Figure 2. Structure of CAVENET.

- The number of lanes and their directions

From the point of view of protocol operations, these parameters can affect the connectivity of the network. In particular, connectivity gaps on a lane can be filled by the presence of relay nodes on the other lanes, as shown in Fig. 1-a. On the other hand, the message penetration on a particular lane can be affected by the radio interference on the opposite lane, as in Fig. 1-b.

- The intersection of lanes

This parameter affect the traffic behaviour on the whole lane, because the crosspoint is the bottleneck for the lane.

Here, we take into account only the first parameter. With respect to the aforementioned properties, we propose to divide the simulator into two blocks, as shown in Fig. 2. The first one, which we call Behavioural Analyzer (BA) block, is concerned with the mobility model, and it should take into account the previous parameters in order to produce accurate mobility traces. The second one, which we name Communication Protocol Simulator (CPS), is the protocol simulator, and it is conceived to test the performance of communication protocols given a particular mobility trace. The BA block should be written in a high-level language, easy to understand and easy to extend. For the particular case of CAVENET, the matrix operations are needed. For this reason, we choose MATLAB. The BA block produces movement patterns which are formatted in a textual format compatible with the CPS's language. Extending the BA block in order to export to other formats is straightforward. The CPS can be one of the many publicly available network simulators, as the well known ns-2 [16]. In principle, the two blocks could also be implemented in two separate machines, in order to speed up the simulation, as in [12].

\section{A. Microscopic Model}

The core of our simulator is 1-dimensional CA model, which has been first studied by Nagel and Schreckenberg (NaS) [17] in a stochastic settings. The CA is a discrete time model of the vehicle traffic. It is governed by three simple rules. However, as for other CAs, these simple rules can well model and reproduce complex real systems. For this reason, the $\mathrm{NaS}$ model has gained a lot of attention during the last ten years.

The time is divided in discrete units $\Delta t$, so that $t_{n}=n \Delta t$. There are $N$ vehicles. A lane $k$ of the road at time $t_{n}, n \in \mathbb{N}$, is represented by a vector $\mathbf{L}_{n}^{k}$ of $L$ sites. The lane is assigned an $N \mathrm{x} 1$ velocity vector $\mathbf{v}_{n}^{k}=\left(v_{i, n}^{k}\right)_{i=1}^{N}$, where $v_{i, n} \in \mathbb{N}_{v_{\max }}$ is the velocity of the vehicle at time $t_{n}$ and position $i$. If the $i$ th site is occupied by a car, $L_{i, n}=v_{i, n}$. Otherwise, $L_{i, n}=-1$. We use the lane index only when it is explicitly required. Every cell or site of the lane has a length of $s$ meters. By setting $v_{\max }=135 \mathrm{~km} / \mathrm{h}$ and $\Delta t=1 \mathrm{~s}$, we obtain $s=7.5 \mathrm{~m}$. At every time step, the velocity $v$ is changed according to the following rules ${ }^{1}$.

Deterministic, $p=0$ or $p=1, \forall i$ :

- 1. $v_{i, n+1}=\min \left(v_{i, n}+1, v_{\max }\right)$

-2. $v_{i, n+1}=\min \left(v_{i, n}, L_{i+1, n}-L_{i, n}-1\right)$

-3. $\mathbf{L}_{n+1}=\mathbf{L}_{n}+\mathbf{v}_{n+1}$

Stochastic:

- $2^{\prime} \cdot v_{n+1, i}=\max \left(0, v_{n, i}-1\right)$, with probability $p$.

The vehicle density is $\rho=N / L$. This simple model can recreate the footprints of real traffic scenarios, such as the $1 / f$ noise of the average velocity observed in real traffic. The dynamics of the systems are regulated by three important parameters, $p, \rho$ and $L$. For example, if $p=0$ the average velocity is $S R D$, otherwise the system present $L_{R D}^{2}$.

\section{B. Improvement of CAVENET}

In the first version of CAVENET, the vehicles were moving in a horizontal line. When a vehicle was at the end of line, in order to continue the simulation we shifted the vehicle at the beginning of line. But, this caused a delay and the vehicles at the beginning and at the end of the line could not communicate with each other. For this reason,

\footnotetext{
${ }^{1}$ We assume parallel update only, i.e. the rules are applied in parallel to every vehicle on the lane.

${ }^{2} \mathrm{~A}$ stochastic process $\left\{X_{n}\right\}_{n=1}^{n=+\infty}$ is SRD if the autocorrelation is summable:

$$
\sum_{k=1}^{+\infty} r(k)<+\infty,
$$

where $r(k)=E\left[\left(X_{n}-\bar{X}\right)\left(X_{n+1}-\bar{X}\right)\right] / \sigma^{2}$. Otherwise, if $r(k)$ is not summable, the process is LRD. This means that very distant samples are not statistically independent, contrary to processes without memory, as the Poisson process which is an SRD process.
} 
we improved the CAVENET, by changing the movement pattern of the vehicles from the straight line to a circle. We also implemented three routing protocols: OLSR, AODV, DYMO and investigated their performance for VANETs.

1) OLSR: The OLSR protocol is a pro-active routing protocol, which builds up a route for data transmission by maintaining a routing table inside every node of the network. The routing table is computed upon the knowledge of topology information, which is exchanged by means of Topology Control (TC) packets.

OLSR makes use of HELLO messages to find its one hop neighbours and its two hop neighbours through their responses. The sender can then select its Multi Point Relays (MPR) based on the one hop node which offer the best routes to the two hop nodes. By this way, the amount of control traffic can be reduced. Each node has also an MPR selector set which enumerates nodes that have selected it as an MPR node. OLSR uses TC messages along with MPR forwarding to disseminate neighbour information throughout the network. Host Network Address (HNA) messages are used by OLSR to disseminate network route advertisements in the same way TC messages advertise host routes.

OLSRv2 is currently being developed at IETF. It maintains many of the key features of the original protocol including MPR selection and dissemination. Key differences are the flexibility and modular design using shared components such as packet format packetbb and neighbourhood discovery protocol.

Recently olsrd has been equipped with the LQ extension, which is a shortest-path algorithm with the average of the packet error rate as metric. This metric is commonly called ETX, which is defined as $\operatorname{ETX}(i)=1 /(N I(i) \times L Q I(i))$. Given a sampling window $W, \mathrm{NI}(i)$ is the packet arrival rate seen by a node on the $i$-th link during $W$. Similarly, LQI $(i)$ is the estimation of the packet arrival rate seen by the neighbour node which uses the $i$-th link. When the link has a low packet error rate, the ETX metric is higher.

2) AODV: The AODV is an improvement of DSDV to on-demand scheme. It minimize the broadcast packet by creating route only when needed. Every node in network maintains the route information table and participate in routing table exchange. When source node wants to send data to the destination node, it first initiates route discovery process. In this process, source node broadcasts Route Request (RREQ) packet to its neighbours. Neighbour nodes which receive RREQ forward the packet to its neighbour nodes. This process continues until RREQ reach to the destination or the node who know the path to destination.

When the intermediate nodes receive RREQ, they record in their tables the address of neighbours, thereby establishing a reverse path. When the node which knows the path to destination or destination node itself receive RREQ, it send back Route Reply (RREP) packet to source node. This RREP packet is transmitted by using reverse path. When the source node receives RREP packet, it can know the path to destination node and it stores the discovered path information in its route table. This is the end of route discovery process. Then, AODV performs route maintenance process. In route maintenance process, each node periodically transmits a Hello message to detect link breakage.

3) DYMO: DYMO is a new reactive (on demand) routing protocol, which is currently developed in the scope of the IETF's MANET working group. DYMO builds upon experience with previous approaches to reactive routing, especially with the routing protocol AODV. It aims at a somewhat simpler design, helping to reduce the system requirements of participating nodes, and simplifying the protocol implementation. DYMO retains proven mechanisms of previously explored routing protocols like the use of sequence numbers to enforce loop freedom. At the same time, DYMO provides enhanced features, such as covering possible MANET-Internet gateway scenarios and implementing path accumulation.

Besides route information about a requested target, a node will also receive information about all intermediate nodes of a newly discovered path. There is a major difference between DYMO and AODV. AODV only generates route table entries for the destination node and the next hop, while DYMO stores routes for each intermediate hop. To efficiently deal with highly dynamic scenarios, links on known routes may be actively monitored, e.g. by using the MANET Neighbourhood Discovery Protocol or by examining feedback obtained from the data link layer. Detected link failures are made known to the MANET by sending a route error message (RERR) to all nodes in range, informing them of all routes that now became unavailable. Should this RERR in turn invalidate any routes known to these nodes, they will again inform all their neighbours by multicasting a RERR containing the routes concerned, thus effectively flooding information about a link breakage through the MANET.

DYMO was also designed with possible future enhancements in mind. It uses a generic MANET packet and message format and offers ways of dealing with unsupported elements in a sensible way.

\section{Vehicle Model}

Every vehicle is a data structure $\mathrm{VE}_{i}$ indexed by its position on the lane. The data structure for the $i$ th vehicle stores: the gap, the velocity, and the current lane position. The relative euclidean position on the lane given by $X_{i}$ is a unique identifier used for the generation of mobility trace. Moreover, for closed boundaries, i.e. if we suppose circular movement of vehicle on the lane, we check if a shift has taken place. This information will serve to properly generate the trace for ns-2. It is straightforward to arrange all these information in a vector form, what is the preferred form used in MATLAB. 


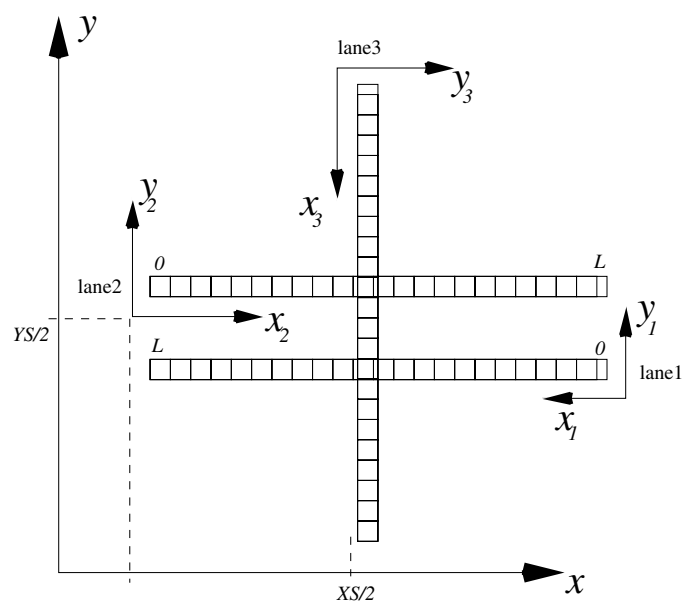

(a)

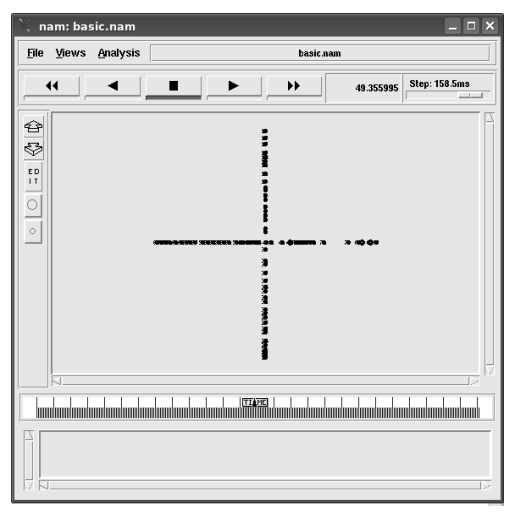

(b)

Figure 3. Lanes construction and ns-2 trace: a) Line construction; b) Excerpt of the generated ns-2 trace for 2 lanes network.

\section{Lane Construction}

Instead of using a particular textual language for describing the position of the lanes in the plane, we use a more general approach. Besides its length, every lane is given a lane transformation, which is used in order to set its real aspect on the plane. This information is used at the mobility trace generation stage. The transformation is a simple affine transformation of the vector $\mathbf{X}_{i}^{k}=\left(X_{i}, Y_{i}, 1\right)$, i.e. the coordinate vector of the $i$ th vehicle on the $k$ th road with respect to the relative reference system. For example, for the lane $\mathbf{L}^{k}$, we have the vehicle structure $\mathrm{VE}_{i}^{k}$. This structure contains the vector $\mathbf{X}_{i}^{k}$. The real position on the plane is computed as $\widetilde{\mathbf{X}}_{i}^{k}=\mathbf{A}(k) \mathbf{X}_{i}^{k}$ where $\mathbf{A}(k)$ is the lane transformation matrix associated with the $k$-th lane, and $\widetilde{\mathbf{X}}_{i}^{k}$ is the vector of coordinates in the absolute reference system (i.e. that used for exporting the ns-2 traces). For example, in Fig. 3, the third lane has the following absolute coordinates:

$$
\widetilde{\mathbf{X}}_{i}^{3}=\left(\begin{array}{ccc}
0 & 1 & \frac{\mathrm{XS}}{2} \\
1 & 0 & \Delta \\
0 & 0 & 1
\end{array}\right)\left(\begin{array}{c}
X_{i} \\
0 \\
1
\end{array}\right)
$$

where XS is the length of the simulation area ${ }^{3}$.

\section{Simulation Results}

\section{A. Mobility Model Validation}

We present here some basic simulations for the $\mathrm{NaS}$ model by means of CAVENET. Hereinafter, we use as simulation variable the average velocity $\bar{v}(t)=N^{-1} \sum_{i=1}^{N} v_{i}(t)=$ $N^{-1}\|\mathbf{v}(\mathbf{t})\|_{1}$ of all cars. CAVENET can analyze and design single and multiple lanes traces. It can also run Monte Carlo simulations. For example, in Fig. 4, we report the results for the so called fundamental diagram, i.e. the flow vs. density diagram. The flow at a particular lane section is defined as $J=\rho \bar{v}$. Each point in the figure is the ensemble

\footnotetext{
${ }^{3}$ The parameter $\Delta$ is used to avoid an apparent bug in ns-2, which fires strange errors when the absolute position is 0 .
}

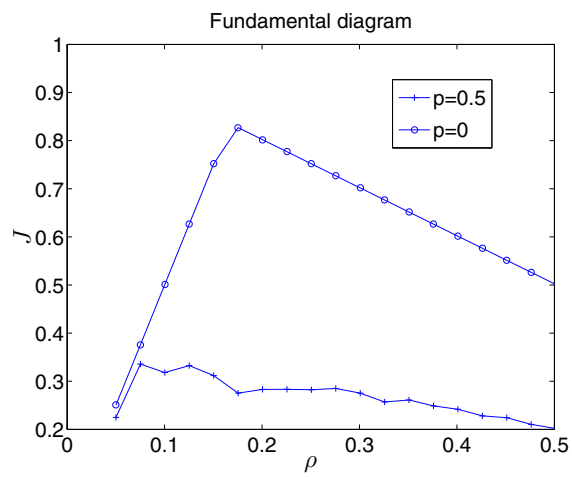

Figure 4. Traffic flow as a function of $\rho$ and $p$ for $L=400$.

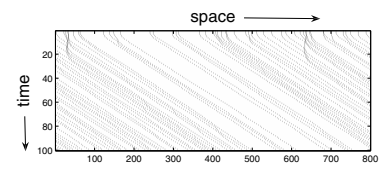

(a) $\rho=0.0625, p=0.3$

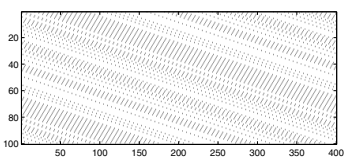

(c) $\rho=0.1, p=0$

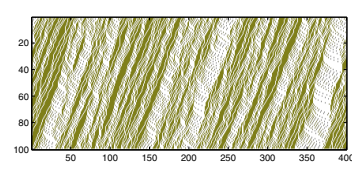

(b) $\rho=0.5, p=0.3$

(d) $\rho=0.5, p=0$

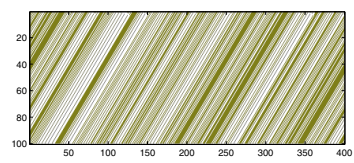

Figure 5. Space-time plots showing the jam wave in different settings.

average over 20 trials of a simulation trace lasting 500 iterations. Moreover, we can also visualize the space-time plot of the traffic, i.e. the evolution of the velocity for every vehicle along the road. We obtain the two traffic regimes, namely the laminar regime and the jammed or congested regime, as shown in Fig. 5-a and Fig. 5-b, respectively. We are interested in the the stationary distribution and transient time, which are very important to assess the next stage simulations, i.e. those related to the communication protocol analysis. 


\section{B. Stationary Distribution}

Usually, RW-like mobility models used in simulation exhibit the velocity decay problem. That means that the simulation variable slowly decays towards a steady state value as the simulation time proceeds. This is problematic, because we do not know when this transient ends. Consequently, we do not know precisely how to remove the transient values. The root of this phenomenon has to be attributed to the underlying mobility model, which has been assumed random. Every node randomly picks a velocity from a continuous uniformly distributed random variable between $\left[v_{\min }, v_{\max }\right]$. The velocity is changed at particular points called waypoints. In this way, the system has an infinite (but countable) number of states. The general solution to this problem consists in finding the steady state distribution of the simulation variable and let the system starts with that distribution. This reasoning is also equivalent to consider Palm probability distributions instead of the usual ones [2].

In our case, the system has inherently a finite state space. The automaton could be represented by a discretetime finite-state Markov chain. We know that a Markov chain with a single class of recurrent states has always a steady state distribution. Moreover, since a Markov chain with finite state space has always at least one recurrent state, we conclude that the steady state distribution exists and is unique. The convergence rate toward this steady-state distribution depends on the eigenstructure of the transition probability matrix of the Markov chain. The problem here is that a Markov chain model is not suitable, because the process can be, in general, LRD, for $0<p<1$. Moreover, even in the SRD case, finding the transition probabilities is not easy.

In general, mobility models for vehicular traffic exhibits a phase transition around a particular value of $\rho$. As we can see in Fig. 5, for $p>0$, the traffic is composed of jammed regions which travel on the opposite direction of movement. For low densities, these waves die out very quickly, as shown also in Fig. 6, but for higher densities there are many interconnected clusters of jammed vehicles. In this case, the steady state is reached very slowly. Therefore, it is important to investigate how many samples should be removed from the staring point in order to sample a process in its stationary regime.

In order to clarify this phenomenon, we measured the transient time $\tau$ for $p=0$, i.e. the deterministic case. In this case, $\bar{v}(t)$ is not LRD. We can show this fact also by plotting the periodogram of $\bar{v}(t)$. In Fig. 7-a, we see that for $f \rightarrow 0$, the periodogram does not diverge. On the other hand, for $p>0$, in Fig. 7-b, the estimated spectrum diverges at the origin, i.e. the underlying process has the LRD property.

\section{Routing Protocols Evaluation}

As evaluation metrics, we use the goodput and Packet Delivery Ratio (PDR). The simulation parameters are shown

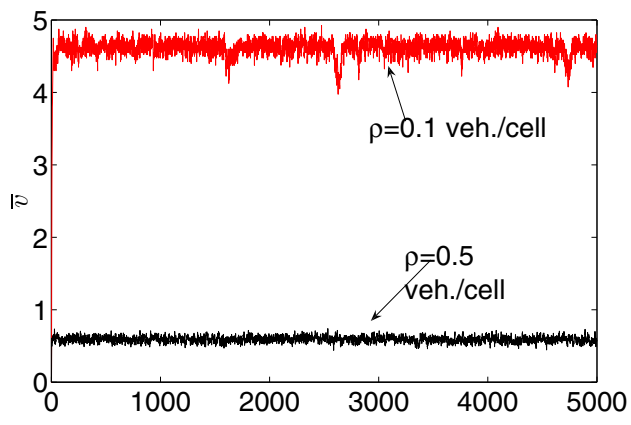

Figure 6. Sample realizations of $\bar{v}(t)$.

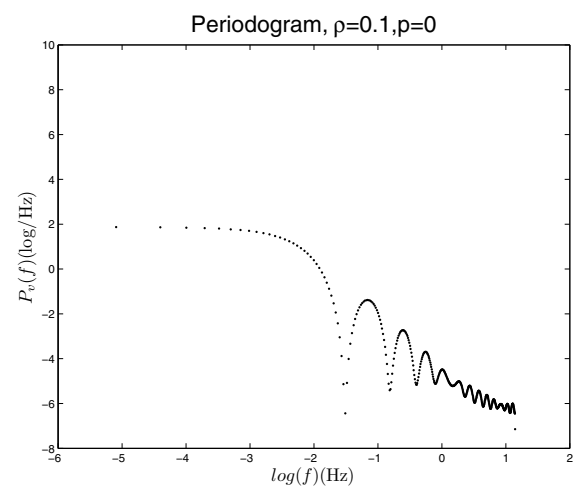

(a)

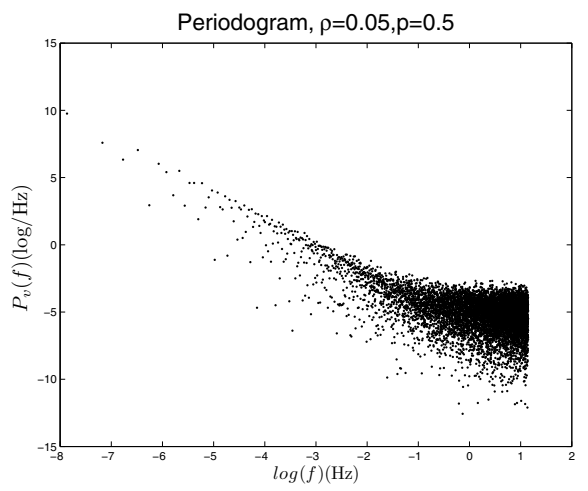

(b)

Figure 7. Deterministic model and stochastic version: a) deterministic model; b) $1 / \mathrm{f}$ noise like spectrum form the the stochastic version of the $\mathrm{NaS}$ model.

in Table 1 . We used one line and 30 nodes for simulations. The simulation time is 100 seconds.

The receiving node is node 0 and the sending nodes are from node 1 to node 8 . We prepared each scenario based on nodes ID. The mobility pattern for all scenarios is the same. In order to evaluate the performance of each protocol, 5 packets per second as a Constant Bit Rate (CBR) traffic were transmitted between 10 seconds and 90 seconds.

The simulations results are shown from Fig. 8 to Fig. 11. In Fig. 8 is shown the goodput of AODV protocol. The goodput of AODV is about ten times of CBR packet size. 
Table I

SIMULATION PARAMETERS.

\begin{tabular}{c|c}
\hline Network Simulator & ns-2 \\
Routing Protocol & AODV, OLSR, DYMO \\
Simulation Time & $100 \mathrm{~s}$ \\
Simulation Area & $3000 \mathrm{~m} \mathrm{Circuit}$ \\
Number of Nodes & 30 \\
Traffic Source/Destination & Deterministic \\
DATA TYPE & CBR \\
Packets Generation Rate & 5 packets/s \\
Packet Size & 512 bytes \\
MAC Protocol & IEEE802.11 DCF \\
MAC Rate & 2 Mbps \\
RTS/CTS & None \\
Transmission Range & $250 \mathrm{~m}$ \\
Radio Propagation Models & Two-ray Ground \\
Hello $A O D V$ Interval & $1 \mathrm{~s}$ \\
Hello $O S R$ Interval & $1 \mathrm{~s}$ \\
TC $O S R$ Interval & $2 \mathrm{~s}$ \\
Hello $D Y M O$ Interval & $1 \mathrm{~s}$ \\
\hline
\end{tabular}

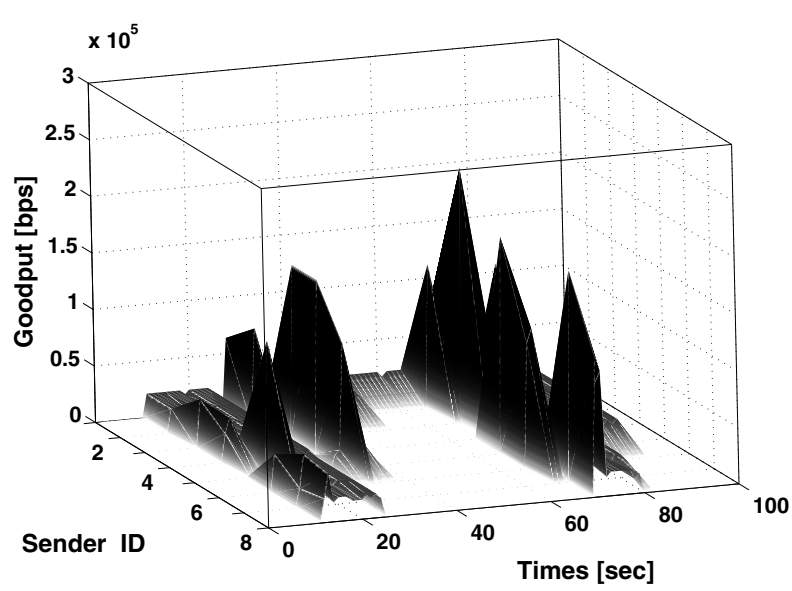

Figure 8. AODV Goodput.

This is because after a back-off time all the accumulated data packets are transmitted in the discovered route. If we increase the background traffic, the number of transmitted packets will again increases and the network may be congested. Also, after 60 seconds, in AODV protocol, there is a delay caused by route finding mechanism. Comparing Fig. 8, Fig. 9 and Fig. 10, we can see that reactive protocols (AODV and DYMO) have better goodput than OLSR. For AODV and DYMO, even the nodes are far from each other they can communicate between 10 seconds to 20 seconds.

In Fig. 11, we show the PDR for three routing protocols. We can see that among three protocols AODV has a better goodput. However, the AODV need more time for searching a new route compared with DYMO. So, the delay of AODV is higher than DYMO. The route searching time of DYMO is almost the same with OLSR protocol. However, DYMO have better goodput than OLSR. Thus, DYMO has a better performance than AODV and OLSR protocols.

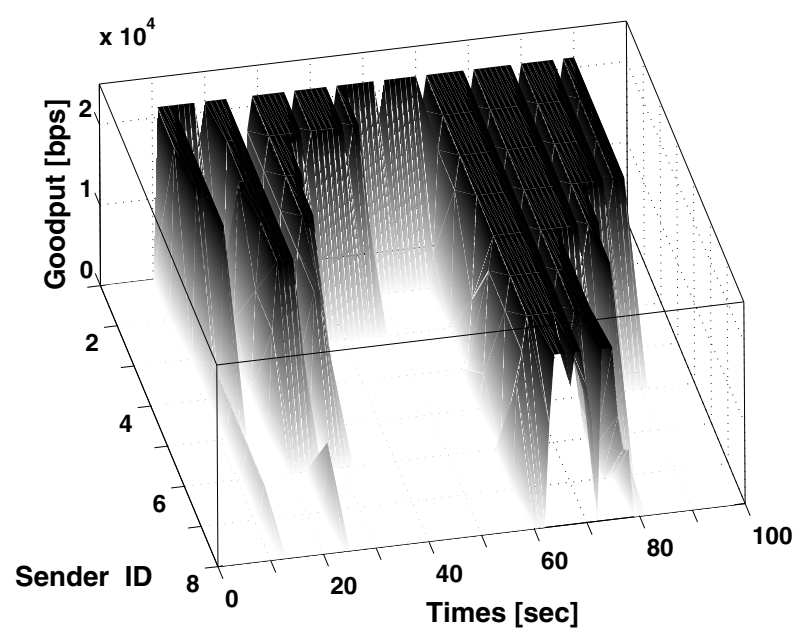

Figure 9. OLSR Goodput.

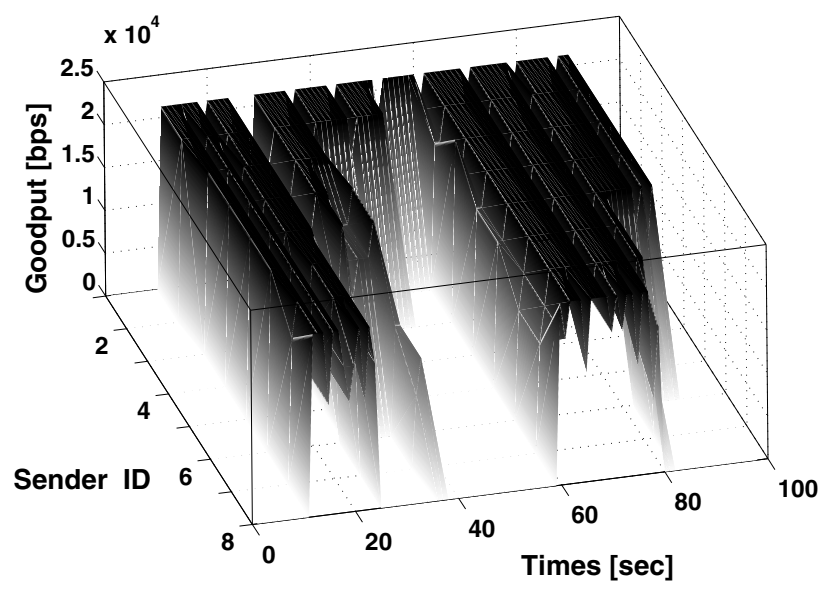

Figure 10. DYMO Goodput.

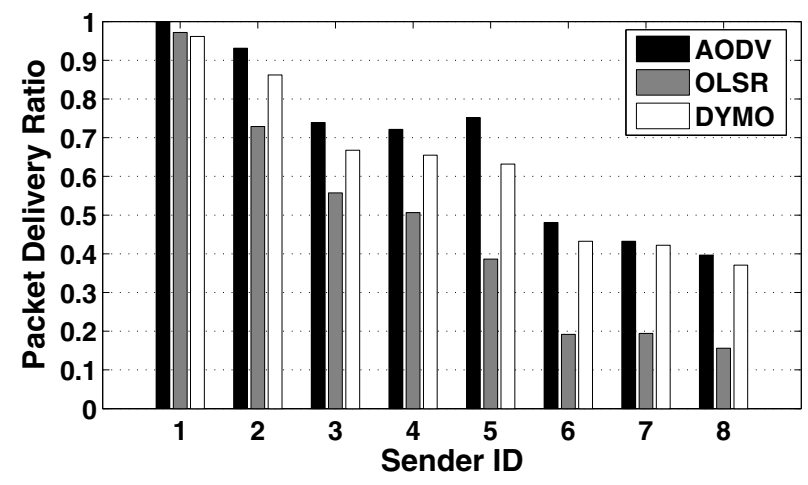

Figure 11. PDR for AODV, OLSR and DYMO.

\section{CONCLUSIONS}

In this paper, we presented CAVENET, a simple simulator for VANETs. The system is modular and it separates mobility from the protocol simulation. 
The mobility model code is written in a language at a level as high as possible, in order to give the researcher a quick understanding of the basic properties of his/her model. For this reason, we used MATLAB.

The basic structure in CAVENET is the vector representing the configuration of a linear lane. The geometry of the lanes is set by affine transformations which are stored in a text file. In such way, the user does not need to learn a particular file format, as in other traffic simulators. By means of CAVENET, we shown some fundamental properties of vehicular traffic which should taken into account when performing network protocols simulations.

We improved the CAVENET by changing the movement pattern of the vehicles from the straight line to a circle and implemented three routing protocols: AODV, OLSR and DYMO. We evaluated the performance of these protocols in VANETs and we found that DYMO has better performance.

In this work, we evaluated AODV, OLSR and DYMO considering goodput and PDR metrics. In the future, we would like to consider other parameters such as routing overhead, traffic quantity and topology change. We also plan to extend our work for different radio propagation modes and environments [18], [19].

\section{ACKNOWLEDGEMENT}

This work is partially supported by a Grant-in-Aid for Scientific Research from Japanese Society for the Promotion of Science (JSPS). The authors would like to thank JSPS for the financial support.

\section{REFERENCES}

[1] J. Harri, F. Filali and C. Bonnet, Mobility Models for Vehicular Ad Hoc Networks: A Survey and Taxonomy, IEEE Communications Surveys \& Tutorials, Vol. 11, No. 4, pp. 19-41, Fourth Quarter 2009.

[2] J. Y. Le Boudec and M. Vojnovic, The Random Trip Model: Stability, Stationary Regime, and Perfect Simulation, IEEE/ACM Transactions on Networking, Vol. 14, No. 6, pp. 1153-1166, 2006.

[3] J. Yoon, M. Liu and B. Noble, A General Framework to Construct Stationary Mobility Models for the Simulation of Mobile Networks, IEEE Transactions on Mobile Computing, Vol. 5, No. 7, pp. 860-871, July 2006.

[4] T. Clausen and P. Jacquet, Optimized Link State Routing Protocol (OLSR), IETF RFC 3626, October 2003.

[5] C. Perkins, E. Belding-Royer and S. Das, Ad hoc OnDemand Distance Vector (AODV) Routing, IETF RFC 3561 (Experimental), July 2003.

[6] I. Chakeres and C. Perkins, Dynamic MANET On-demand (DYMO) Routing, Internet Draft (draft-ietf-manet-dymo-14), June 2008, Work in progress.
[7] C. Sommer and F. Dressler, The DYMO Routing Protocol in VANET Scenarios, In Proc. of 66-th IEEE Vehicular Technology Conference (VTC2007-Fall), pp.16-20, 2007.

[8] F. Bai, N. Sadagopan, and A. Helmy, IMPORTANT: A Framework to Systematically Analyze the Impact of Mobility on Performance of Routing Protocols for Ad-hoc Networks, In Proc. of IEEE INFOCOM-2003, pp. 825-835, MarchApril 2003.

[9] N. Sadagopan, F. Bai, B. Krishnamachari, and A. Helmy, PATHS: Analysis of Path Duration Statistics and Their Impact on Reactive MANET Routing Protocols, In MobiHoc2003: Proc. of the 4-th ACM International Symposium on Mobile Ad Hoc Networking \& Computing, pp. 245-256, 2003.

[10] M. Fiore, J. Harri, F. Filali, and C. Bonnet, Vehicular Mobility Simulation for VANETs, Proc. of the 40-th Annual Simulation Symposium (ANSS-2007), pp. 301-309, 2007.

[11] L. Smith, R. Beckan, R. Anson, K. Nagel, and M. Williams, TRANSIMS: Transportation Analysis and Simulation System, In Proc. of the 5-th National Transportation Planning Methods Applications Conference, LA-UR 95-1664, April 1995.

[12] M. Piorkowski, M. Raya, A. L. Lugo, M. Grossglauser, and J. P. Hubaux, Joint Traffic and Network Simulator for VANETs, In Proc. of Mobile and Information Communication Systems Conference (MICS-2006), October 2006, Available on line at: http://www.mics.ch/.

[13] G. De Marco, M. Tadauchi and L. Barolli, Description and Analysis of a Toolbox for Vehicular Networks Simulation, In Proc. of IEEE ICPADS/PMAC-2WN-2007, Vol.2, pp.16, 2007.

[14] J. Haerri, F. Filali and C. Bonnet, Performance Comparison of AODV and OLSR in VANETs Urban Environments under Realistic Mobility Patterns, In Proc. of 5th IFIP Mediterranean Ad-Hoc Networking Workshop (Med-HocNet-2006), Lipari, Italy, 2006.

[15] J. Haemi, M. Fiore, F. Filali and C. Bonnet, A Realistic Mobility Simulator for Vehicular Ad Hoc Networks, EURECOM Technical Report, 2007 Available at: http://www.eurecom.fr/util/publidownload.en.htm?id=1811.

[16] Information Science Institute (ISI), Network Simulator Version 2 (NS-2), http://www.isi.edu/nsnam.

[17] K. Nagel and M. Schreckenberg, A Cellular Automaton Model for Freeway Traffic, Journal of Physics I France, Vol. 2, pp. 2221-2229, December 1992.

[18] L. Barolli, Gj. Mino, F. Xhafa, G. De Marco, A. Durresi, A. Koyama, Analysis of Ad-Hoc Networks Connectivity Considering Shadowing Radio Model, In Proc. of MoMM2009, Kuala Lumpur, Malaysia, pp. 464-468, December 2009.

[19] D. Dhoutaut, A. Régis and F. Spies, Impact of Radio Propagation Models in Vehicular Ad Hoc Networks Simulations, In Proc. VANET-2006, pp.40-49, September 2006. 